\title{
First Monte Carlo simulation study of Galeras volcano structure using muon tomography
}

\author{
A. Tapia ${ }^{* a}$, D. Dueñas ${ }^{b}$, J. Rodriguez $^{b}$, J. Betancourt ${ }^{b}$ and D.A. Martinez Caicedo ${ }^{c}$ \\ ${ }^{a}$ Departamento de Ciencias Básicas, Universidad de Medellín, Medellín, Colombia. \\ ${ }^{b}$ Universidad de Nariño, Pasto, Colombia. \\ ${ }^{c}$ IIT Center for Accelerator and Particle Physics, Illinois Institute of Technology, Chicago-IL \\ (USA). \\ E-mail: atapia@udem.edu.co, dafra9oegmail.com, \\ jairo3584@gmail.com, dmarti36eiit.edu
}

\begin{abstract}
Muon radiography is based on the observation of the absorption of muons in matter, as the ordinary radiography does by using X-rays. The interaction of cosmic rays with the atmosphere produce Extensive Air Showers (EAS), which provide abundant source of muons. These particles can be used for various applications of muon radiography, in particular to study the internal structure of different volcanoes edifice. We will focus on Galeras volcano located $9 \mathrm{~km}$ from Pasto city (Colombia).

In this work we present the first study of the muon lateral distribution at Pasto altitude (4276 $m$ a.s.l.) and a preliminary simulation the volcanic cone using GEANT4[1]. For the interaction of the cosmic rays with the atmosphere we have used CORSIKA 74004[2] software with an atmosphere tropical model and QGSJETII-04[3] as hadronic model for the high energies and GHEISHA2002d[4] for low energies. The analysis considers two different primary particle (proton and iron), four zenith angles $\left(0^{\circ}, 30^{\circ}, 45^{\circ}\right.$ and $\left.60^{\circ}\right)$ with energies values of 5,10 and 100 $\mathrm{TeV}$.
\end{abstract}

38th International Conference on High Energy Physics 3-10 August 2016

Chicago, USA

${ }^{*}$ Speaker. 


\section{Introduction}

The Galeras Volcano with a height of 4276 m.a.s.l., located in Pasto city (Colombia) with an estimated age of 4.500 years, is one with the highest activity in Colombia with important records of eruptions in the past. The increasing of population in higher risk areas around the volcano has motivated to develop special techniques of monitoring the volcano activity, especially for its records respecting to pyroplastic flux generated in previous volcano eruption. The tomography with atmospheric muons help us to understand the internal volcano structure and its magma dinamics during a eruptive process[5].

EAS simulation using CORSIKA software has been implemented to obtain the lateral distribution of atmospheric muons (MLDF) and their energy spectrum when they arrive at the altitude of Pasto city. Furthermore, a preliminary simulation of the interaction of these muons with Galeras volcano has been done using GEANT4[1]. The geometric shape of Galeras volcano was performed with SOLiDWORKS (SW) software and implemented for GDML in FASTRAD.

\section{Methodology}

The EAS were simulated with four different zenith angles: $\theta=0^{\circ}, 30^{\circ}, 45^{\circ}$ and $60^{\circ}$; two types of primary particles (proton and Fe) with energies of 5, 10, $100 \mathrm{TeV}$; QGSJEII-04[3] as the hadronic interaction model for high energies and GHEISHA2002d[4] for low energies. We have used the CORSIKA's tropical atmospheric model and Pasto city magnetic field components Bx and Bz were generated with the NOAA geomagnetic calculator[6].

The GEANT4 toolkit was used to simulate the interaction of muons within volcano and its crater. The geometry has been constructed using SW and converted to GDML files. As input in SW contours provided by the volcano observatory "Servicio Geologico Colombiano (SGC)" were used[7]. The volcano chemical composition had the following percentage: O (46.6\%), $\mathrm{Si}(27,7 \%), \mathrm{Al}$ $(8.3 \%)$ and $\mathrm{Fe}, \mathrm{Ca}, \mathrm{Na}, \mathrm{K}, \mathrm{Mg}(17.4 \%)$. The volcano geometry was definied using a crater diameter of $320 \mathrm{~m}$, depth of $250 \mathrm{~m}$ and a height of $1100 \mathrm{~m}$.

\section{Results}

Using CORSIKA were simulated 200 EAS for two type of primary particles (Proton and Fe) and angles and energies previously described. For proton as primary particle in figure 1 are shown the MLDF (left) and the number of muons arriving to the altitude of Pasto city ( $2600 \mathrm{~m}$ ) in function of energy (right).

The MLDF obtained from simulation behaves according Nishimura-Kamata-Greizen equation[8] for the KASCADE-Grande[9] detectors array:

$$
\rho_{\mu}=N_{\mu}\left(\frac{r}{r_{0}}\right)^{-\alpha}\left(1+\frac{r}{r_{0}}\right)^{-\beta}\left(1+\left(\frac{r}{10 r_{0}}\right)^{2}\right)^{-\gamma},
$$

where $N_{\mu}, r_{0}, \alpha, \beta$, and $\gamma$ are fit parameters. These parameters $r_{0}, \alpha$, and $\gamma$ were fixed in $320 \mathrm{~m}$, 0.75 and 3 respectively[11]. In this analysis the fitted parameters are $\beta$ and the scaling factor $N_{\mu}$ of the distributions. The values of the $N_{\mu}$ and $\beta$ fit parameters are presented in Table 1 for Proton as 

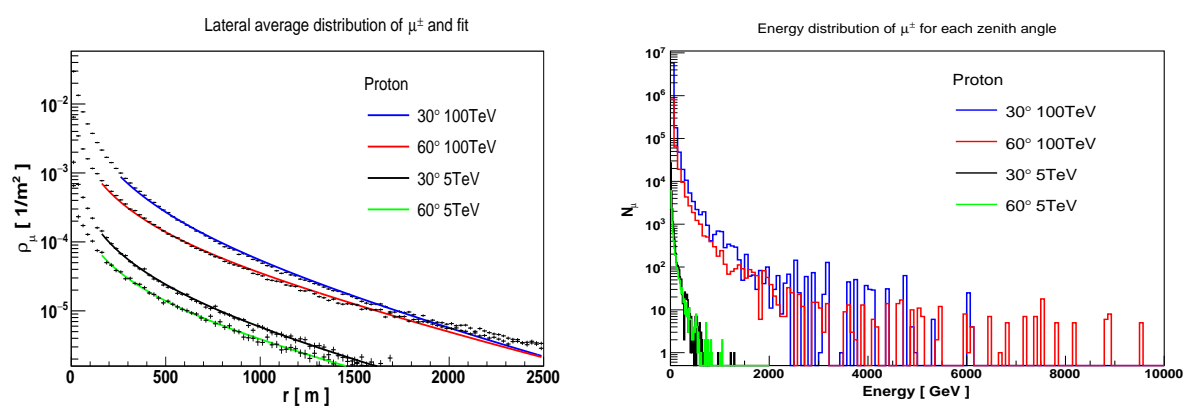

Figure 1: Number of muons per $m^{2}$ (left) and energy spectra of muons expected in surface (right) for proton as primary particle. Fe results are similar and could be find at Ref. [10].

primary particle, different zenith angles and primary energy. For Fe case the fit parameters follow the same tendency.

\begin{tabular}{|c|c|c|c|c|c|c|c|c|c|}
\hline \multicolumn{10}{|c|}{ Proton } \\
\hline \multirow{5}{*}{$5 \mathrm{TeV}$} & & $N_{\mu}$ & $\beta$ & \multirow{5}{*}{$10 \mathrm{TeV}$} & $N_{\mu}$ & $\beta$ & \multirow{5}{*}{$100 \mathrm{TeV}$} & $N_{\mu}$ & $\beta$ \\
\hline & $0^{\circ}$ & 0.00014 & 1.5 & & 0.00025 & 1.5 & & 0.0029 & 2.0 \\
\hline & $30^{\circ}$ & 0.00014 & 1.4 & & 0.00021 & 1.4 & & 0.0023 & 1.9 \\
\hline & $45^{\circ}$ & 0.000097 & 1.3 & & 0.00015 & 1.3 & & 0.0015 & 1.6 \\
\hline & $60^{\circ}$ & 0.000063 & 1.2 & & 0.000010 & 1.1 & & 0.00074 & 1.3 \\
\hline
\end{tabular}

Table 1: Parameters obtained from fitting the number of muons per square meter as a function of core distance using equation 3.1 .

\subsection{Preliminar results of volcano simulation using GEANT4}

In GEANT4 besides the volcano geometry simulation implementation as shown in figure 2 (top-right), we have simulated 1000 muons with energy of $1 \mathrm{TeV}$ passing through the volcano crater. We made a study giving to the crater two types of composition: standard rock and air. Results from this study are shown in figure 2 (bottom).

As a first approach we send the particles through the volcano geometry in an scaled 1:1000. This scaling was done due a simulation at real scale will need an increasing computational power. We are in the process to obtain acces to a cluster and scale the simulation to the real dimensions of the volcano.

\section{Conclusions}

We have estimated for the first time the MLD at the Pasto city altitude with different zenith angles and energy of the primary particles of the EAS.

We have developed the first steps through a complete simulation of the Galeras volcano geometry, and volcano composition using GDML files as input into GEANT4.

Future steps include calculate the atmospheric profile for Pasto city using stored data in the Global Data Assimilation System (GDAS) platform. Furthermore we are planning to improve the 


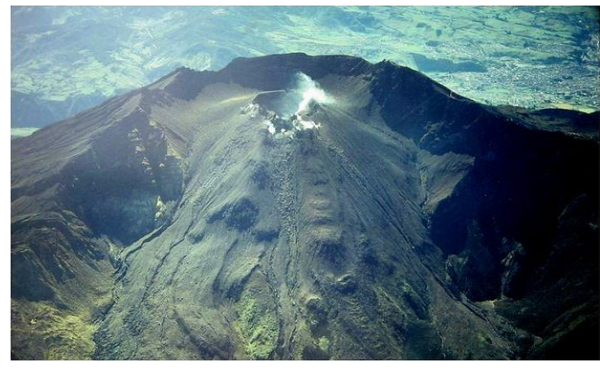

Interactions Air Crater

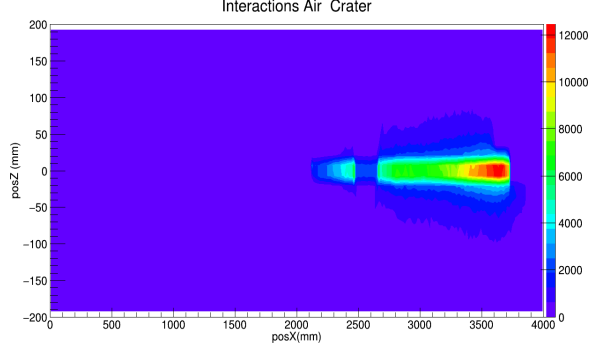

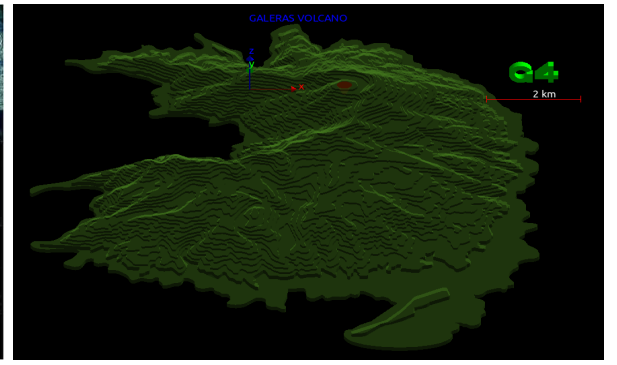

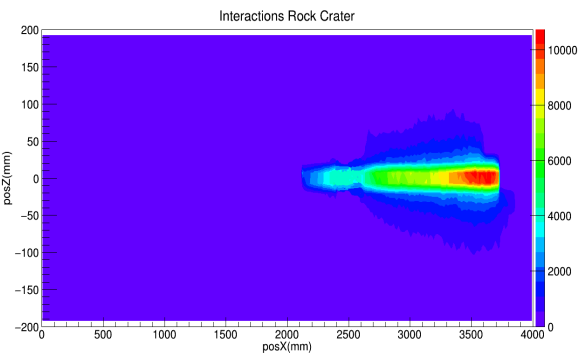

Figure 2: Real picture (top left) and GEANT4 simulation of the Galeras volcano (top right). Interaction of 1000 muons with the volcano crater (bottom). Muons of $1 \mathrm{TeV}$ and two types of crater composition were taken into consideration: air (bottom-left) and standard rock (bottom-right).

simulation of Galeras volcano composition and increase the number of muons interaction in the volcano using GEANT4 and the real dimensions of it accesing to a cluster. Finally we are in our first steps developing simulations of the particle detectors using plastic scintillator bars and SiPM as one of the future candidates for readout of the particle detector to be constructed once all the simulations are complete.

\section{References}

[1] J. Allison et al, IEEE Transactions on Nuclear Science 53 No. 1 (2006) 270-278 and S. Agostinelli et al, Nuclear Instruments and Methods A 506 (2003) 250-303.

[2] D. Heck et al, CORSIKA Vol. 6019 FZKA (1998).

[3] S. Ostapchenko, Physical Review D 83 (2011) 014018.

[4] H.C. Fesefeldt, Technical Report PITHA (1985) 85-02.

[5] H. K. M. Tanaka et al, Nat. Commun. 5:3381 (2014).

[6] NOAA National Centers for Environmental Information, http://www.ngdc.noaa.gov/geomag-web/.

[7] Servicio Geológico Colombiano, Observatorio Vulcanológico y Sismológico de Pasto, http://www2.sgc.gov.co/Pasto/Volcanes/Volcan-Galeras/Generalidades.aspx.

[8] K. Kamata, J. Nishimura, Prog. Theoret. Phys. Suppl. 6 (1958) 93 and K. Greisen, Ann. Rev. Nucl. Sci. 10 (1960) 63.

[9] KASCADE-Grande Collab, in proceedings of $29^{\text {th }}$ ICRC 6 (2005) 301.

[10] 38 ${ }^{\text {th }}$ ICHEP poster contribution, https://indico.cern.ch/event/432527/contributions/1071873/.

[11] A. Tapia et al, AAA Workshop 7 (2015) 237-240 [astro-ph. HE/1501 . 02217]. 